\title{
Extreme adaptive optics simulations for EPICS
}

\author{
Visa Korkiakoski ${ }^{\mathrm{a}}$ and Christophe Verinaud \\ Laboratoire d'Astrophysique, Orservatoire de Grenoble, BP 53, F-38041 Grenoble Cedex 9, France
}

\begin{abstract}
We show end-to-end extreme adaptive optics (XAO) simulation results for EPICS (Exo-Planets Imaging Camera and Spectrograph). The impacts of telescope jitter, spiders and photon noise are analysed. In addition, the stroke demand for XAO deformable mirror (DM) is studied and wavefront sensor choice discussed. Simulation results indicate that current baseline design meets the EPICS requirements.
\end{abstract}

\section{Introduction}

The EPICS project is currently in the conceptual design study phase. The central science case of EPICS is the direct imaging and characterization of exoplanets seen in reflected light. Requirements of intensity contrast in Near Infrared between exoplanet and host star are $10^{-8}$ at 30 mas angular separation and $10^{-9}$ at 100 mas on bright stars. Extreme AO is necessary to obtain a low halo of scattered light permitting the detection of exoplanets through spectroscopy and polarimetry.

The huge diameter of $42 \mathrm{~m}$ primary of E-ELT presents significant challenges. The XAO system has to control $\sim 30000$ actuators, which means computational demands approaching the borders of conceivable technologies: a command matrix in a traditional matrix-vector-multiplication (MVM) reconstruction has a size of $13 \mathrm{~GB}$. In addition, the wavefront sensing at ELTs, in particular in high contrast applications, favours non-linear and less known wavefront sensors (WFSs) capable of detecting accurately low spatial frequencies.

This work presents the first end-to-end simulation results of AO for EPICS. At first, the baseline design is illustrated (2). Then, we characterize the impacts of telescope jitter and spiders (Sect. 3 and 4). Finally, Sect. 5 gives an analysis of stroke-contrast trade-off in the presence of the error sources.

\section{EPICS AO system}

The current baseline for EPICS AO system is to use two cascaded and independent AO loops as illustrated in Fig. 1. The first loop is a classical single conjugate (SCAO) system using the E-ELT wavefront correction, M4 and M5, for low-order and tip/tilt correction. It needs a WFS having a large dynamical range due to telescope jitter creating large tip/tilt wavefront errors that needs to be measured. The second loop uses a low-stroke XAO DM and a WFS dedicated for high contrast applications.

We have done our simulations assuming the 1 st stage WFS has a sampling of $80 \times 80$ and sensing is done at $0.6 \mu \mathrm{m}$. The 2 nd stage sampling is $200 \times 200$ and sensing is done at $0.825 \mu \mathrm{m}$. First loop operates at $1 \mathrm{kHz}$, the second at $3 \mathrm{kHz}$. Wavefront reconstruction is a traditional matrix-vectormultiplication. We use both zonal and pseudo-Fourier modes (no difference in performance). Controllers are simple integrators with tip/tilt having independently optimized loop gains. The other simulation parameters are shown in Table 1.

Due to ease of simulation, we have restricted our WFS consideration to three Foucault-style sensors, known for having interesting properties for XAO on ELTs [1]: classical pyramid sensor [2]

\footnotetext{
a e-mail: vkorkiak@obs.ujf-grenoble.fr
} 


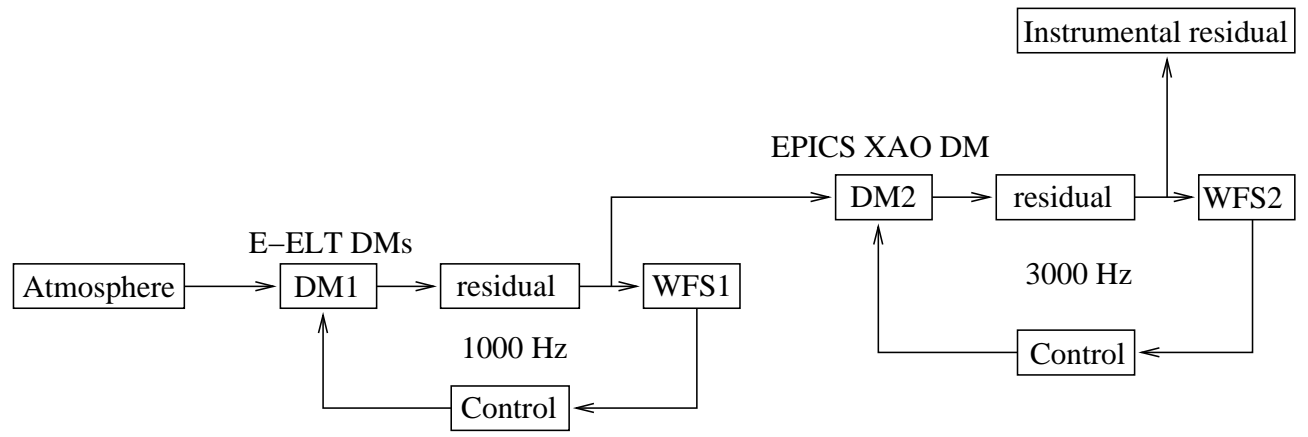

Fig. 1. Schema of EPICS AO system.

Table 1. Simulation parameters.

\begin{tabular}{ll|ll}
\hline Telescope diameter & $42 \mathrm{~m}$ & $\lambda_{\mathrm{OBS}}$ & $1.35 \mu \mathrm{m}$ \\
Central obstruction & $12.6 \mathrm{~m}$ & $\lambda_{\text {WFS }}$ (telescope AO) & $0.6 \mu \mathrm{m}$ \\
frame-rate (telescope AO) & $1 \mathrm{kHz}$ & $\lambda_{\text {WFS }}$ (EPICS XAO & $0.825 \mu \mathrm{m}$ \\
frame-rate (EPICS XAO) & $3 \mathrm{kHz}$ & Influence functions & Piezoelectric, $~ 20 \%$ coupling \\
WFS sampling (telescope AO) & $80 \times 80$ & Actuators (telescope AO) & 4788 \\
WFS sampling (EPICS XAO) & $200 \times 200$ & Actuators (EPICS XAO) & 29120 \\
\hline Number of layers & 3 & Layer heights & $10 \mathrm{~m}, 1 \mathrm{~km}, 10 \mathrm{~km}$ \\
Wind speeds (m/s) & $10,15,20$ & Layer weights & $20 \%, 65 \%, 15 \%$ \\
Outer scale $L_{0}$ & $26 \mathrm{~m}$ & $r_{0}$ at $0.5 \mu \mathrm{m}$ & $0.125 \mathrm{~m}(\mathrm{GS}$ direction) \\
\hline Number of time steps & 1024 & Number of pixels/pupil & $1600 \times 1600$ \\
\hline
\end{tabular}

(PWFS), roof sensor (RS) and modified optical differentiation sensor (MODS). See Fig. 2 for illustration. The pyramid sensors, classical PWFS and RS, have shown to have excellent sensitivity, but low dynamical range [3]. The classical PWFS has less optical components compared to RS, but it suffers from diffraction effects deteriorating its performance [4]. The third sensor, MODS, has been introduced to increase the dynamical range of pyramid sensors while maintaining many beneficial properties of PWFS.

Optical differentiation sensor has been presented as a good XAO sensor [5], and we have slightly modified the concept. MODS is similar to roof sensor, except that the prisms are replaced by partially reflective/transmissive masks. The middle part of the mask has a linearly changing profile causing the signal measure the phase gradient at low spatial frequencies. The outer part of the mask is flat meaning the sensor's characteristics at high spatial frequencies resemble those of the roof sensor. The dynamical range of MODS can be chosen by the width of the linear mask region (we studied widths of $2 \alpha=6-40 \lambda / D)$.

Our simulations indicate that using the classical PWFS on E-ELT might be difficult due to its diffraction effects. The pyramid prism causes diffraction at the detector plane in four directions, as illustrated in Fig. 3. At the presence of large central obstruction, these diffraction effects cause local loss of sensitivity at certain places of pupil. In closed loop operation this results in symmetrical "spikes" (increased error in residual phase) near the obstruction as shown in Fig. 3. Hundreds of iterative corrections are required to remove the spikes, even in moderate seeing conditions and $3 \mathrm{kHz}$ correction rate.

The diffraction phenomenon appears even when excluding all sub-beam interferences and is independent of used DM actuators. The only way to avoid these problems, as far as we know, is to use two roof prisms instead of a single pyramid prism.

A non-modulated RS works well in a regime of low wavefront distortions, but has too low dynamical range for 1st stage WFS. A MODS does well at the 1st stage, but is not able to detect well the low spatial frequencies, which is crucial for XAO sensor at the presence of spiders (see Sect. 4). 


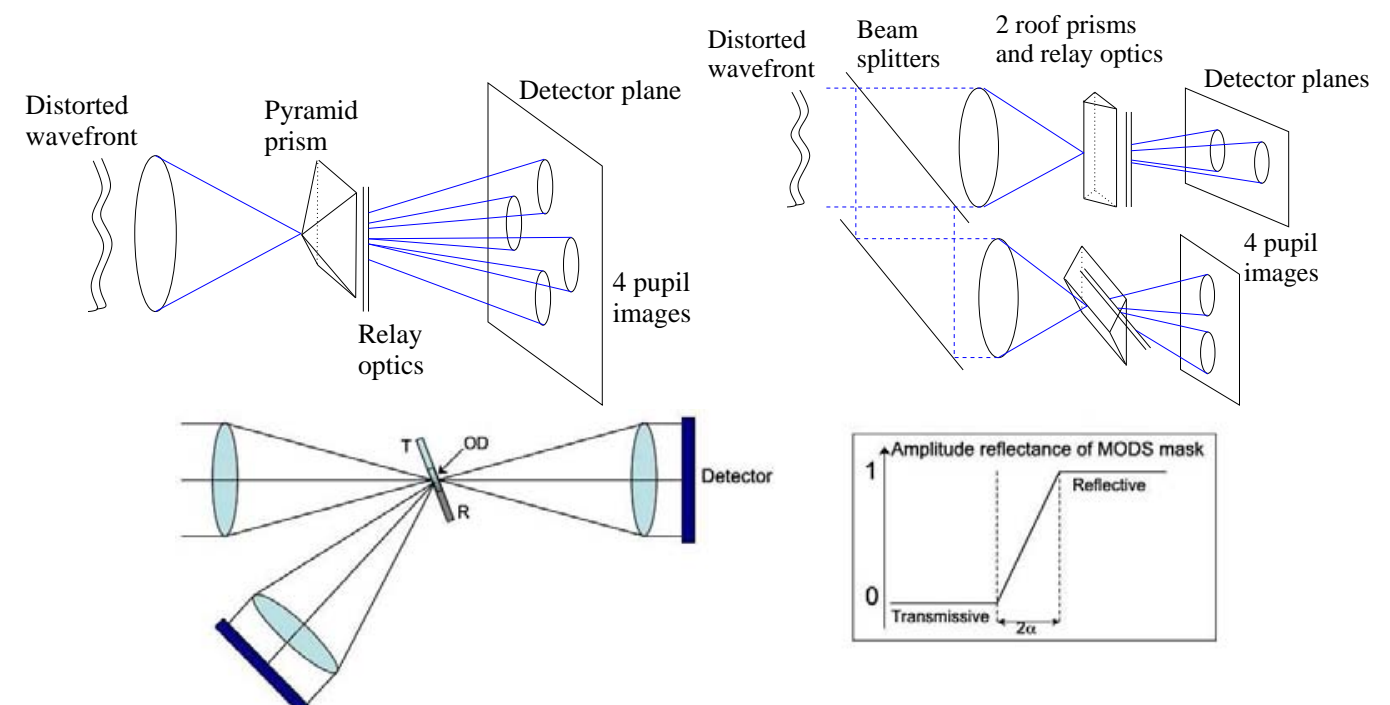

Fig. 2. Illustrations of three Foucault-type WFSs. Upper row: classical four-sided PWFS and roof sensor. Lower row: modified optical differentiation sensor with a ramp width of $2 \alpha$.
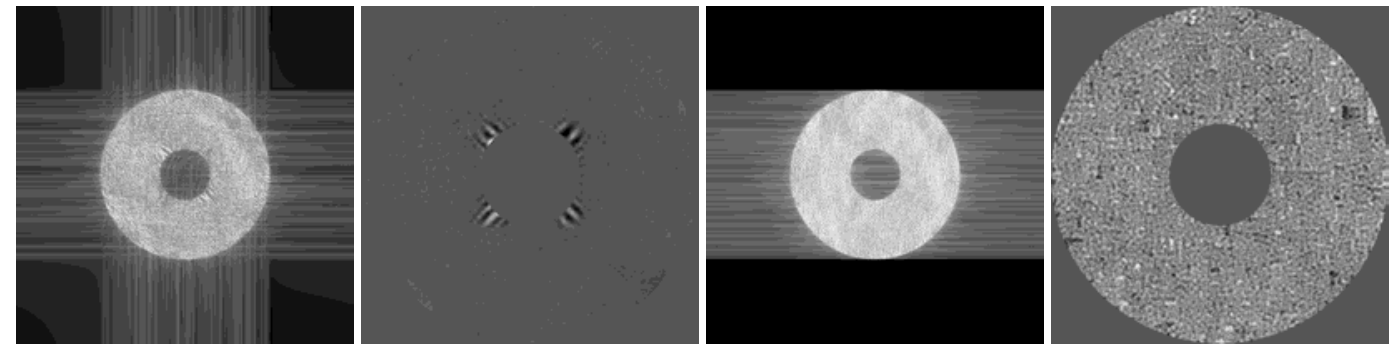

Fig. 3. Illustration of PWFS diffraction effects. Images are scaled independently. 1st and 3rd image: intensities of one pupil image at detector plane (non-linear scales), 4-sided PWFS left and RS right. 2nd and 4th image: the resulting closed loop residuals.

\section{Impact of telescope jitter and noise}

Telescope jitter due to wind load is a significant error source in AO. It is seen even at the time scales of less than a second as a slowly evolving tip/tilt component on the atmospheric phase. In our simulations we have taken one of the most pessimistic case presented by the E-ELT telescope group: we change the tilt component from 0.05 to 0.22 arcsec during the simulation run (duration $0.3 \mathrm{~s}$ ).

The impact of jitter and photon noise on the final raw contrast is illustrated in Fig. 4. It shows the radially averaged contrast for four simulations with different error sources and presents also an analytic estimate for comparisons.

It is seen that with only temporal error present, the simulated contrast is an order of magnitude better than an analytic estimate [6]. This is due to pessimistic assumptions in the estimate. The WFS error (red plot) is 2-5 times in contrast compared to perfect actuator fitting (yellow plot).

Telescope jitter increases contrast only at spatial frequencies less than 20 mas, and thus does not affect the scientific observations. The photon noise, however, can increase the contrast significantly at spatial frequencies $>100$ mas. With a flux of $\sim 10^{7}$ photons $/ \mathrm{m}^{2} / \mathrm{s}$ (detected photons in the WFS bandwidth), the loss in contrast is at worst about an order of magnitude. This flux corresponds to $V=7$ G star (Solar type), which is EPICS requirement for limiting magnitude for the detection of mature gas giant planets. In the simulations we share the bandwidth such that 2 nd stage WFS receives 10 times more photons compared to the 1 st WFS. This ratio can be optimized later. 


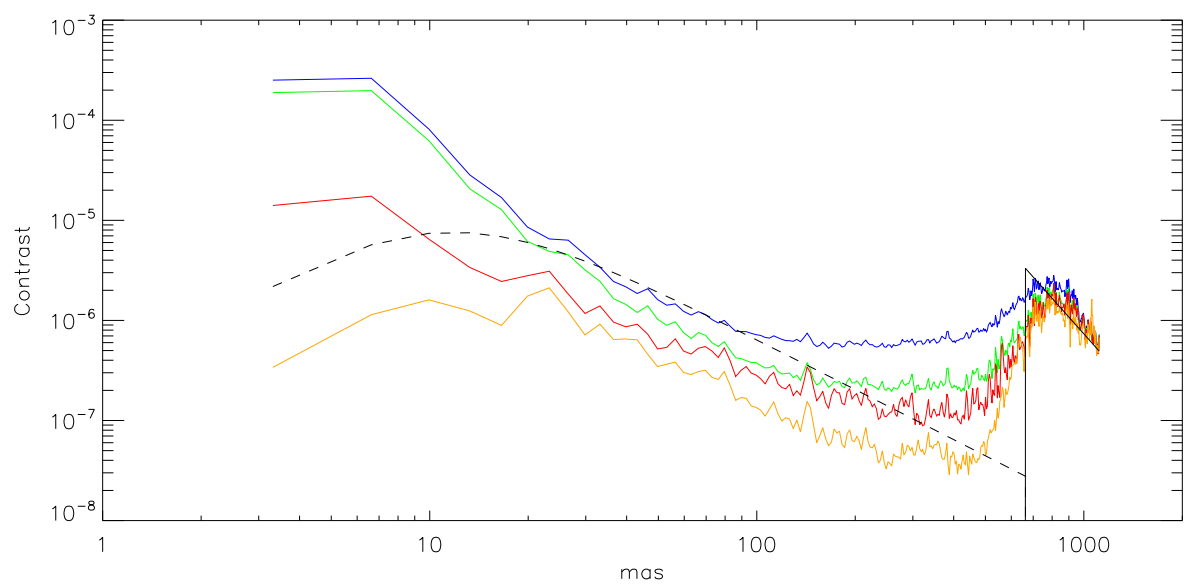

Fig. 4. Radially averaged contrasts after XAO correction. Orange: perfect actuator fitting (only temporal error present). Red: only WFS error (no photon noise nor jitter). Green: telescope jitter, photon flux of $10^{8}$ photons $/ \mathrm{m}^{2} / \mathrm{s}$. Blue: telescope jitter, photon flux of $10^{7}$ photons $/ \mathrm{m}^{2} / \mathrm{s}$. The fluxes are detected photons in the WFS bandwidth.

\section{Issues with spiders}

The support structures of secondary mirror will split the phase into separated islands. We have simulated spiders having a width of $50 \mathrm{~cm}$ and shape as shown in Fig. 5. We observed the spiders causing significant effects that can be, however, rather efficiently compensated with a proper choice of wavefront sensors and reconstruction and control methods.

To deal with the spiders, we realized that profoundly different approaches are needed for the $1 \mathrm{st}$ stage SCAO and the 2nd stage XAO systems.

At the 1st stage, the spiders are hiding "sub-apertures" only partially. In addition, in closed loop operation, the WFS is used with high aberrations while calibration is done in diffraction limited conditions. At the second stage, however, several "sub-apertures" are completely masked by spiders and phase discontinuity is complete. XAO is also able to correct much more turbulence. That makes the diffraction effects of the spiders, induced by a Foucault-type WFS, more prominent also in closed loop operation on-sky.

We found that best performance of the 1st stage SCAO system was obtained when its calibration (interaction matrix computation) was done without using spiders. No modifications were done to the closed loop operation. We believe the turbulence still present after SCAO correction smears the diffraction effects caused by spiders, and thus the calibration should be done in conditions where those effects are not visible.

The performance loss after SCAO was found to be from $\sim 0.80$ to $\sim 0.75$ in terms of Strehl ratio at $2.2 \mu \mathrm{m}$, when using a MODS with a ramp width of $40 \lambda / D$. This decrease is due to the loss in sensitivity when detecting the isolated piston modes (see the closed loop residual phase left in Fig. 5). A roof sensor would detect the piston modes better (since it is sensitive to low spatial frequencies), but it would saturate easier.

The 2nd stage XAO confronts different problems. The total phase discontinuity at spiders can lead to situation where the XAO DM corrects the separated islands well, but leaves them apart of an integer number of sensing wavelengths. However, we found it easy to avoid by starting XAO correction only after the 1st stage AO loop has been closed and correcting only the low spatial frequencies during the first $\sim 100$ time steps.

It is also important to use a sensor extremely sensitive to low spatial frequencies. If we use MODS (or apply modulation), the isolated piston modes are not corrected well. In addition, when using the non-linear roof sensor, it is important that the diffraction effects of spiders are taken into account at the calibration stage — otherwise reconstruction errors are made near the spiders. 
The three different attempts to use XAO with spiders are illustrated in Fig. 5. The 1st image shows the optimal closed loop residual phase after SCAO. The 2nd image shows what happens with MODS or RS with modulation: the low order "island modes" are poorly detected. The 3rd image shows what happens when RS is used without including spiders in the calibration: correction is done imperfectly near the spiders (those errors decrease the contrast 1-2 order of magnitudes). The 4th image is the working solution: performance is almost the same as without spiders. Thus, the XAO is able to compensate the performance loss at the 1st stage, the cost being increased stroke demand (see Sect. 5).
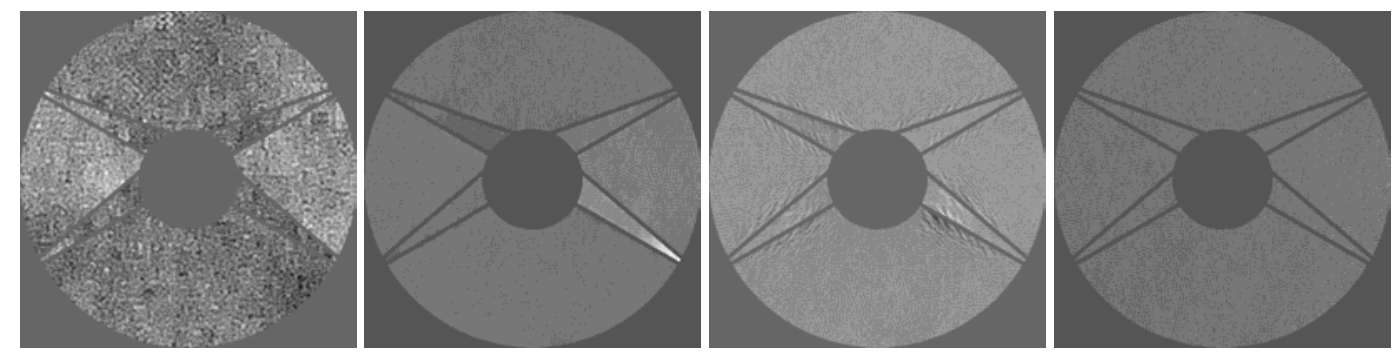

Fig. 5. Closed loop residual phases from simulations with spiders. 1st: only SCAO correction (MODS, ramp $40 \lambda / D$ ). 2nd: XAO WFS is MODS (ramp $6 \lambda / D$ ). 3rd: XAO WFS is RS, calibration made without spiders. 4th: XAO WFS is RS, calibration made with spiders.

Although this work demonstrates that the E-ELT spiders do not pose dramatic problems for XAO, a few significant issues remain. One difficult question to be addressed is the implementation of fast reconstruction algorithms. The simulations here show that a decent performance might not be achieved, if the full diffraction effects of roof prisms and spiders are not included in the reconstruction models. All currently developed fast reconstruction methods are developed for linear and non-diffractive sensors like Shack-Hartmann. In addition, the rotation of the spiders during observation requires modifications to the reconstruction parameters. In the case of traditional MVM reconstruction, the 13 GB command matrix should be updated at certain intervals, whose length is to be studied.

\section{Stroke versus contrast}

The cascaded AO system of two independent closed loops means that the amount of correction needs to be balanced between the DMs. If the stroke at the XAO DM is critical, as much turbulence as possible must be corrected with E-ELT M4 and M5. However, the choice of high loop gains for SCAO can propagate noise and decrease the final contrast. Thus, a tradeoff between the XAO DM stroke and obtained contrast must be done.

We have done simulations to illustrate the issue. The instrumental PSD is computed using several controller parameters (loop gains) and XAO DM stroke is recorded. The results are shown in Fig. 6. The contrast is plotted as a function of XAO DM stroke when controller parameters of 1st AO loop are modified. Several noise levels are simulated and jitter is always present.

It is seen that the general stroke requirement for XAO $(1.5-2 \mu \mathrm{m}$ in the studied cases) is 3-4 times smaller than DM specification. The contribution of the spiders on the stroke demand is $\sim 0.25 \mu \mathrm{m}$. In addition, the stoke-contrast tradeoff is easy at fluxes $\geq 10^{7} \mathrm{ph} / \mathrm{m}^{2} / \mathrm{s}$ : the SCAO gains can be set as high as needed without significantly propagating noise. At fluxes $\leq 10^{6} \mathrm{ph} / \mathrm{m}^{2} / \mathrm{s}$ only slight noise propagation issues emerges: the loss in contrast is increased at most by a factor of 2 , if XAO DM stroke is limited.

\section{Conclusions}

The first end-to-end XAO simulation results for EPICS are presented. The intensity contrast after twostage AO correction and XAO DM stroke are studied in the presence of the major error sources: WFS 


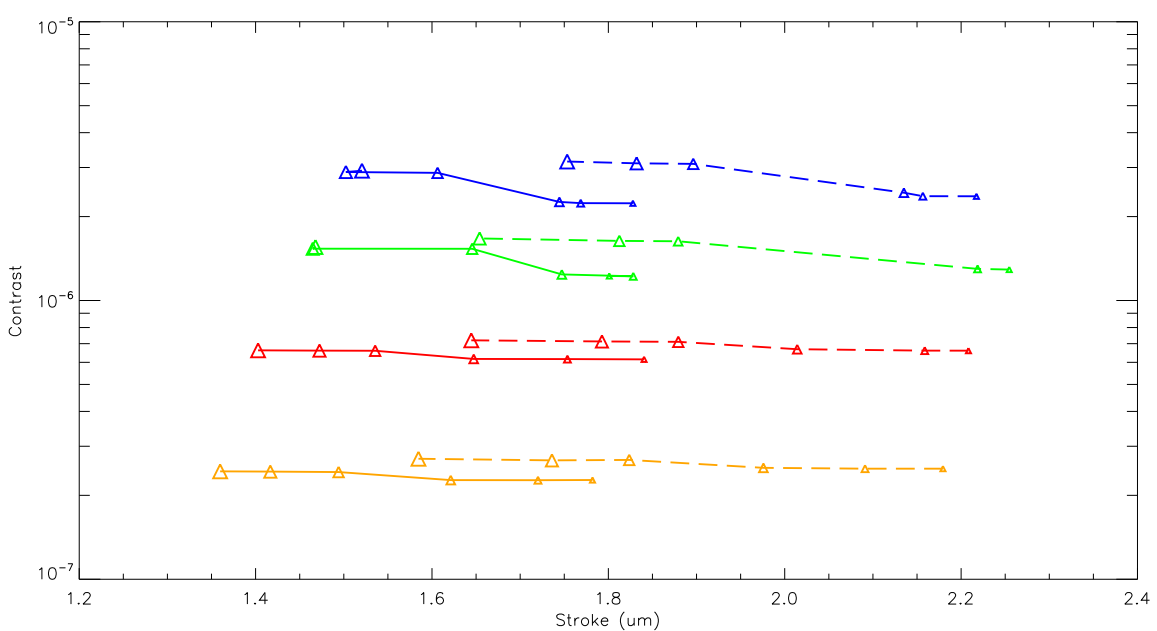

Fig. 6. Contrast as a function of XAO DM stroke when loop gains are modified. We use an average contrast, computed over radial distances of $20-80 \%$ of the corrected field. The stroke is an optimistic optical stroke estimate. It is computed such that $0.2 \%$ of the most extreme DM shape values are excluded, and the stoke is the difference of the remaining extreme values. The gain values for the 1st stage are 1.6-4.0 for tip/tilt, 0.8-1.6 for other modes. The plots show only the optimized gains for the 2nd stage (0.8-1.2). Dashed plots are from simulations with spiders, solid plots show cases without spiders. The colors show different photon fluxes: from bottom to down $10^{8}, 10^{7}, 10^{6}, 10^{5}$ and $5 \cdot 10^{5}$ photons $/ \mathrm{m}^{2} / \mathrm{s}$ in the same way as in Sect. 3.

non-linearity, temporal error, photon noise, telescope jitter and spiders. A raw contrast (after XAO correction and a coronagraph) of $10^{-5}$ is reached at 20 mas and $10^{-7}-10^{-6}$ at $200-500$ mas. Needed optical stroke for XAO DM $(1.5-2 \mu \mathrm{m})$ is well below current specifications.

Telescope jitter seem to be well corrected by the $1 \mathrm{kHz} 1$ st stage loop. However, if its correction is slower, the stroke requirements for XAO DM will increase. Impacts of spiders can be well compensated by XAO, when a non-modulated roof sensor is used.

WFS requirements have been more specified. The 1st WFS need to have characteristics like MODS to operate well at large dynamical range, but avoid noise propagation at higher spatial frequencies. The 2nd WFS needs to detect low spatial frequencies extremely well to correct the spider induced island modes. Thus, only little modulation is possible.

Significant challenges are foreseen for fast reconstruction algorithms: non-modulated roof sensor has non-sparse command matrix and cannot be modelled as a filtering operation in Fourier space. It remains to be studied whether additional WFSs or other solutions are needed.

\section{References}

1. O. Guyon, The Astrophysical Journal629, 592 (2005)

2. R. Ragazzoni, Journal of Modern Optics 43, 289 (1996)

3. C. Vérinaud, Optics Communications 233, 27 (2004)

4. V. Korkiakoski, C. Vérinaud, M. Le Louarn, R. Conan, Applied Optics46, 6176 (2007)

5. J.E. Oti, V.F. Canales, M.P. Cagigal, Mon. Not. R. Astron. Soc.360, 1448 (2005)

6. F.J. Rigaut, J.P. Veran, O. Lai, Analytical model for Shack-Hartmann-based adaptive optics systems (1998), Vol. 3353 of Proc. SPIE, pp. 1038-1048

Acknowledgement: our numerical simulations have been performed using clusters at the CIMENT platform maintained by Joseph-Fourier University at Grenoble. 